\title{
Editorial
}

\section{Esclerosis múltiple: abandonemos la rutina}

\author{
Nicolás Sgarbi ${ }^{1}$ \\ ${ }^{1}$ Exprofesor Agregado del Departamento Clínico de Radiología, \\ Médico Colaborador Calificado Honorario de Neurocirugía del \\ Instituto de Neurología, Hospital de Clínicas, Montevideo, Uruguay
}

Rev Argent Radiol 2021;85:55-56.

La esclerosis múltiple (EM) es una enfermedad inflamatoria crónica, desmielinizante y degenerativa del sistema nervioso central, causa más común, después del trauma craneoencefálico, de discapacidad en la población joven. Tiene alta prevalencia en América Latina y es motivo frecuente de realización de estudios de resonancia magnética (RM).

El trabajo realizado por Herazo-Bustos y col. ${ }^{1}$ nos plantea la necesidad de evaluar el valor de la RM y cómo impacta la utilización de algunas secuencias en el diagnóstico de esta frecuente enfermedad cuando existe compromiso medular.

El valor de la RM ha sido claramente establecido, tanto para el diagnóstico como para el seguimiento, control de tratamiento y pronóstico, siendo fundamental en la toma de decisiones.

El diagnóstico se fundamenta en la presencia de lesiones de sustancia blanca que cumplan con los criterios clásicos de diseminación en tiempo y espacio, ambos basados en la clínica y los hallazgos de imagen. ${ }^{2}$

Numerosas pautas y consensos han establecido los requerimientos mínimos de los protocolos de estudio y los intervalos adecuados entre ellos, siendo fundamental la estandarización. ${ }^{3}$

En los últimos años, se han propuesto cambios que tienen un impacto significativo en la evaluación de los pacientes, aunque su introducción en la práctica es muy variable.

Las secuencias ponderadas en 3D FLAIR se imponen como la herramienta más importante para la evaluación de la carga lesional, hallazgo de nuevas lesiones y valoración de respuesta al tratamiento. ${ }^{3}$

También se propone la utilización selectiva de gadolinio, analizando caso a caso los posibles aportes de este, y teniendo presentes sus posibles efectos a largo plazo. ${ }^{3}$

Se discute la importancia de utilizar equipos de alto campo, pero no se han demostrado diferencias estadísticamente significativas con equipos de $1.5 \mathrm{~T}$ de uso rutinario, $\mathrm{y}$ solo se recomiendan si están disponibles.
El estudio de la médula espinal para el diagnóstico de EM es parte fundamental de los criterios de McDonald de 2017, por la alta incidencia de lesiones medulares en pacientes con sindrome clínico aislado. ${ }^{2}$

En pacientes con síndrome radiológico aislado, la presencia de lesiones medulares sin traducción clínica se ve en poco más del 65\% de los que luego desarrollan EM, y en el 100\% de las formas primariamente progresivas. ${ }^{4}$

Para un adecuado estudio medular, se recomienda utilizar secuencias en el plano sagital, habitualmente fast spin echo T2 (FSE), short tau inversion recovery (STIR) o densidad protónica (DP), siendo opcional el plano axial.

Se ha demostrado que no es suficiente con utilizar secuencias ponderadas en FSE T2 por su baja sensibilidad global, por lo que es fundamental el complemento con otra de las secuencias recomendadas, sobre todo la DP.

Las secuencias DP son superiores en la detección de lesiones medulares independiente del segmento estudiado, con cerca de un $30 \%$ más de lesiones observadas sin aumento de los falsos positivos. ${ }^{1,5,6}$

Como ya mencionamos, la detección de lesiones medulares es de gran importancia en los pacientes afectados de EM, no solo por sus aportes al diagnóstico y eventuales diferenciales, sino también por su impacto en el pronóstico.

La afectación medular traduce fenómenos desmielinizantes, pérdida neuronal, destrucción axonal y gliosis, lo que produce una disfunción múltiple, sensitiva, motora y auntonómica. ${ }^{4}$

De igual forma que a nivel cerebral, se debe evaluar la carga lesional medular y la presencia de atrofia por su impacto pronóstico negativo.

La atrofia medular es mas significativa en pacientes con EM progresiva que en aquellas formas con empujes y remisiones, correlacionándose con el grado de discapacidad. ${ }^{7,8}$

Sin dudas, la realización de RM en pacientes con EM debe ser personalizada, dado que cada uno tiene un fenotipo

(C) 2021. Asociación Civil Sociedad Argentina de Radiología and Federacion Argentina de Asociaciones de Radiología, Diagnóstico por Imágenes y Terapia Radiante. All rights reserved.

This is an open access article published by Thieme under the terms of the Creative Commons Attribution-NonDerivative-NonCommercial-License, permitting copying and reproduction so long as the original work is given appropriate credit. Contents may not be used for commercial purposes, or adapted, remixed, transformed or built upon. (https://creativecommons.org/ licenses/by-nc-nd/4.0/)

Thieme Revinter Publicações Ltda., Rua do Matoso 170, Rio de Janeiro, RJ, CEP 20270-135, Brazil 
específico de enfermedad, con un pronóstico diferente, por lo cual es de gran importancia la cabal comprensión de los aportes de la RM y sus distintos biomarcadores. ${ }^{9}$

\section{References}

1 Herazo-Bustos C, Tramontini Jens C, Mora Salazar JA, Nieto Taborda $\mathrm{K}$, Navas C. Comparación entre las secuencias axiales de densidad de protones y $\mathrm{T} 2$ en lesiones medulares cervicales de pacientes con esclerosis múltiple. Rev Argent Radiol 2021;85(03):57-61

2 Hartung HP, Graf J, Aktas O, Mares J, Barnett MH. Diagnosis of multiple sclerosis: revisions of the McDonald criteria 2017 continuity and change. Curr Opin Neurol 2019;32(03):327-337

3 Wattjes MP, Ciccarello O, Reich DS, Banwell B, de Stefano N, Enzinger C, et al. Magnetic Resonance Imaging in Multiple Sclerosis study group Consortium of Multiple Sclerosis Centres North American Imaging in Multiple Sclerosis Cooperative MRI guidelines working group. 2021 MAGNIMS-CMSC-NAIMS consensus recommendations on the use of MRI in patients with multiple sclerosis. Lancet Neurol. 2021;20(08):653-670
4 Moccia M, Ruggieri S, Ianniello A, Toosy A, Pozzilli C, Ciccarelli O. Advances in spinal cord imaging in multiple sclerosis. Ther Adv Neurol Disord 2019;12:1756286419840593

5 Chong AL, Chandra RV. Chuah, Roberts EL, Stuckey SL. Proton density MRI increases detection of cervical spinal apinal cord multiple sclerosis lesions compared with T2-weighted Fast SpinEcho. AJNR Am J Neuroradiol. 2016;37(01):180-184

6 Karavasilis E, Velonakis G, Argiropoulos G, et al. Proton density fat suppressed MRI in 3T increases the sensitivity of multiple sclerosis lesion detection in the cervical spinal cord. Clin Neuroradiol. 2019;29(01):45-50

7 Leguy S, Combès B, Bannier E, Kerbrat A. Prognostic value of spinal cord MRI in multiple sclerosis patients. Rev Neurol (Paris). 2021; 177(05):571-581

8 Rocca MA, Preziosa P, Filippi M. What role should spinal cord MRI take in the future of multiple sclerosis surveillance? Expert Rev Neurother. 2020;20(08):783-797

9 Chen Y, Haacke EM, Bernitsas E. Imaging of the spinal cord in multiple sclerosis: past, present, future. Brain Sci. 2020;10(11): 857 